\title{
DYNAMICS AND STABILITY OF TWO POWER GRIDS WITH HUB CLUSTER TOPOLOGIES
}

\author{
Vladislav Khramenkov \\ Nonlinear Dynamics Department \\ Institute of Applied Physics RAS \\ Russia \\ hramenkov.vlad@appl.sci-nnov.ru
}

\author{
Aleksei Dmitrichev \\ Nonlinear Dynamics Department \\ Institute of Applied Physics RAS \\ Russia \\ admitry@appl.sci-nnov.ru
}

\author{
Vladimir Nekorkin \\ Nonlinear Dynamics Department \\ Institute of Applied Physics RAS \\ Russia \\ vnekorkin@appl.sci-nnov.ru
}

Article history:

Received 20.05.2019, Accepted 11.06.2019

\begin{abstract}
We report the results of study of two models of power grids with hub cluster topology based on the secondorder Kuramoto system. The first model considered is the small grid consisting of a consumer and two generators. The second model is the Nizhny Novgorod power grid. The areas in the parameter spaces of the grids that corresponds to different modes, including working synchronous one, of their operation are obtained. The dynamic stability of synchronous mode in the Nizhny Novgorod power grid model to transient disturbances of the power at its elements is tested. We show that the stability of peripheral elements of the grid to disturbances depends significantly on the lengths of their connections to the rest of the grid.
\end{abstract}

\section{Key words}

Power grids, the Nizhny Novgorod power grid, Kuramoto system, synchronization, transient stability.

\section{Introduction}

The power grid is a part of the energy system in which electrical energy is produced, converted, transmitted and consumed. Successful operation of the grid largely depends on its ability to provide reliable and uninterrupted power supply to consumers. One of the main requirements for ensuring reliable operation of the grid is the preservation of synchronization of electrical power generators during consumed-power dis- turbance, power-plant failures, spurious actuations of the automatic-protection systems in the electric-power transmission systems and their breaks, as well as some other events. The loss of synchronization among the generators of the grid can provoke cascading failures and lead to disintegration of the grid into individual clusters (power islands) and eventually to blackouts. The clusters are formed around large-scale power plants such as nuclear or hydroelectric power plants, etc., which represent the so-called hubs i.e. the core elements of the grids whose number of couplings significantly exceeds the average number of couplings of other elements. Thus, it is not only necessary to study the stability of synchronous modes of the grids itself, but also of their individual hub clusters. The basic element of the grid is a synchronous machine that can work as a generator or a consumer. The dynamics of a synchronous machine is described by the Park-Gorev equations [Gorev, 1959; Park, 1929]. But these equations are complex enough to apply them to large-scale power grids and a variaty of different approaches are used. Among them topology oriented approach which applies the findings of graph theory and different measures introduced on the graphs of the grids to their statical analyses [Pagani and Aiello, 2013; Dwivedi and Yu, 2013; Song et al., 2017], probabilistic approach based on the construction of probabilistic models for studying of statistical patterns of development of cascade failures [Solé et al., 2008; Rosas-Casals, 2010] and dynamical approach based on findings and methods of theory of dynamical systems [Hill and Guanrong Chen, 2006; Witthaut and Timme, 2012; Lozano et al., 2012; Motter et al., 2013; Menck et al., 2014; 
Klinshov et al., 2015; Filatrella et al., 2008; Drfler and Bullo, 2011; Drfler and Bullo, 2012; Drfler et al., 2012; Grzybowski et al., 2018; Arinushkin and Anishchenko, 2018]. Widely recognized dynamical approach assumes that the power grid is a set of interconnected synchronous machines that simulate the operation of generators and consumers of electrical energy. The state of each machine is determined from the equation of motion of its rotating part - the rotor. The quantity characterizing this state is the phase of the rotor in a coordinate system rotating with the reference frequency of the network. The dynamics of the elements of the grid is described by the second-order Kuramoto model:

$$
\ddot{\theta}_{i}=P_{i}-\alpha \dot{\theta}_{i}+\sum_{j=1}^{N} k_{i, j} \sin \left(\theta_{j}-\theta_{i}\right)
$$

In equations (1), $\theta_{i}$ is the rotor phase of $i$ th machine, $N$ is the number of machines in the grid. Parameter $P_{i}$ characterizes either input or output mechanical power of the electrical machine; it is negative for consumers and positive for generators. The term $-\alpha \dot{\theta}_{i}$ characterizes the power loss and $\alpha$ is the damping coefficient. The term $k_{i, j} \sin \left(\theta_{j}-\theta_{i}\right)$ characterizes electrical power transmitted between the $i$ th and $j$ th grid elements; $k_{i, j}$ is a maximal power of transmission line between these elements, and besides $k_{i, j}=k_{j, i}$. The constancy of this term in time determines the synchronous (working) mode of operation of the power grid.

In the paper we numerically investigate dynamics and transient stability of two models of power grids with hub cluster topologies. The first model is simple enough and consists of a consumer and two generators. In [Dmitrichev et al., 2017] we studied the model analytically and established that for certain values of parameters it has four equilibrium states one of which is stable and corresponds to synchronous mode of the grid and the others are saddle ones. Here we prove that there is a region of global asymptotic stability of the synchronous mode. The second model is the Nizhny Novgorod power grid. The topology of this model is more complex and consists of several hubs. We study the dynamic stability of synchronous mode of the model to transient disturbances of the power.

\section{Results}

First we consider a small hub cluster grid with topology shown in Figure 1 consisting of a consumer and two generators. Assuming $k_{1,2}=k_{1,3}=K$ the dynamics of the grid would be described by the following system:

$$
\left\{\begin{array}{l}
\ddot{\varphi}_{1}=-\gamma_{1}-\lambda \dot{\varphi}_{1}-2 \sin \left(\varphi_{1}\right)-\sin \left(\varphi_{2}\right) \\
\ddot{\varphi}_{2}=-\gamma_{2}-\lambda \dot{\varphi}_{2}-\sin \left(\varphi_{1}\right)-2 \sin \left(\varphi_{2}\right)
\end{array}\right.
$$

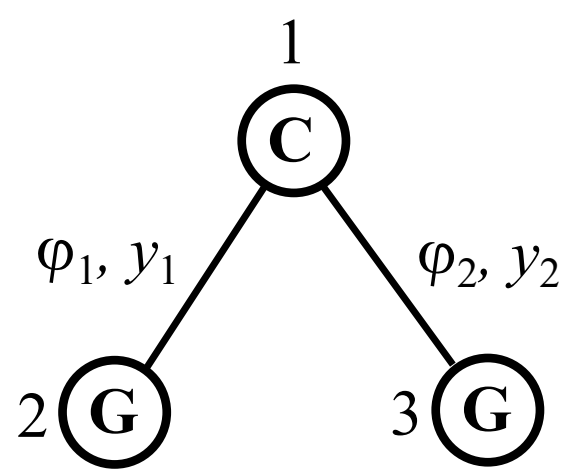

Figure 1. Topology of the hub cluster power grid model with a consumer and two generators.

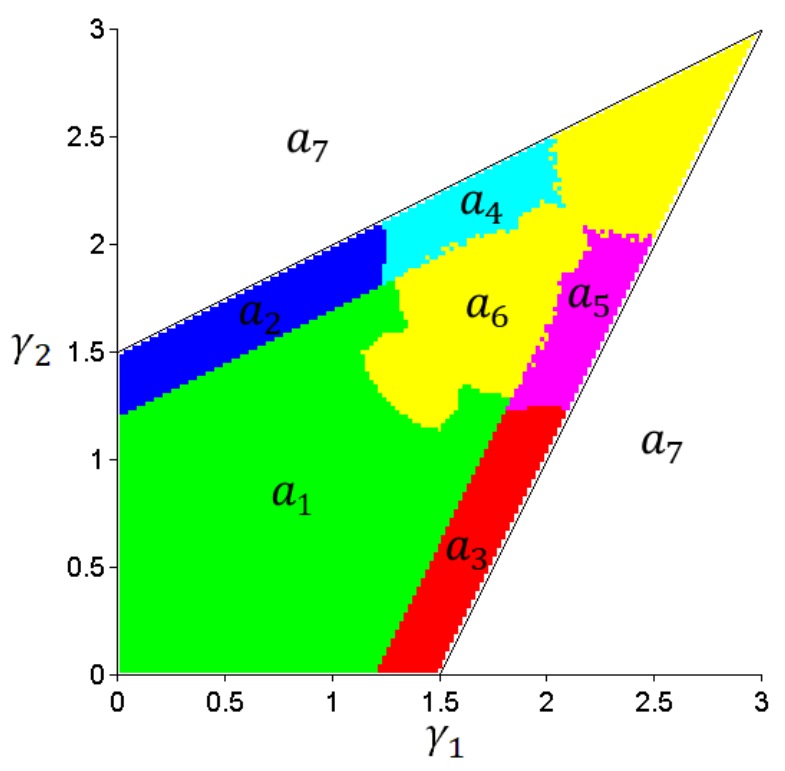

Figure 2. Partition of $\left(\gamma_{1}, \gamma_{2}\right)$-parameter plane on regions with different modes of the small hub cluster grid. Parameter values: $\lambda=$ 0.7 .

In equation (2), $\varphi_{i}=\theta_{i+1}-\theta_{1}$ are the phase differences between the generators and the consumer; parameters $\gamma_{i}=\frac{P_{1}-\widetilde{P}_{i+1}}{K}$ are proportional to the difference between the mechanical powers of the consumer and the generators; parameter $\lambda=\frac{\alpha}{\sqrt{K}}$.

By using numerical and analytical (Lyapunov functions, comparison systems) methods, a partition of parameters plane into the areas corresponding to different modes of operation (a synchronous mode, a quasisynchronous mode, an asynchronous mode and combinations of these modes) of the small hub grid is obtained (see Figure 2).

In area $a_{1}$ there is only synchronous mode. At the synchronous mode, the variables $\varphi_{1}$ and $\varphi_{2}$ are constant in time. In areas $a_{2}$ and $a_{3}$ there are synchronous mode and two types of quasi-synchronous modes. At quasisynchronous mode one of the variables $\varphi_{i}$ oscillates in 
time around some average value and another variable decrease or increase in time (see Figure 3a). In areas $a_{4}$ and $a_{5}$ there are synchronous mode, two types of quasi-synchronous modes and asynchronous mode. At asynchronous mode both variables $\varphi_{1}$ and $\varphi_{2}$ decrease or increase in time (see Figure $3 \mathrm{~b}$ ). In area $a_{6}$ there are synchronous mode and asynchronous mode. In area $a_{7}$ there is no synchronous mode.

Next we consider hub cluster grid that reproduces the basic properties of the Nizhny Novgorod power grid, based on a data from [Ministry of Energy of the Russian Federation, 2014]. The corresponding topologies of real and model power grids are shown in Figure 4.

Here we assume that $k_{i, j}=1 / l_{i, j}$, where $l_{i, j}$ is the length of the transmission line, between the $i$ th and $j$ th elements. Also $P_{i}=P_{g}$ for generator elements and $P_{i}=-\frac{P_{g} N_{g}}{N-N_{g}}$ for consumers, where $P_{g}$ is the power of a generator in the grid and $N_{g}$ is the number of generators. In that case the power balance condition $\left(\sum P_{i}=0\right)$ is automatically performed and synchronous mode can exist for appropriate choice of parameters. We found the parameters for which there is the synchronous mode in the grid. Then we investigated dynamic stability of the synchronous mode to transient disturbances of the power at the elements of the grid in the form of a rectangular pulse of duration $\tau$ and amplitude $\Delta P_{i}$. Figure 5 shows the intervals of safe power disturbances at the generators and consumers. In this intervals (initial) synchronous mode of the grid recovers after action of perturbation ends. Notice that 1 th and 2 th peripheral elements are least stable to power disturbances.

We studied in detail the dependence of the magnitude of the safe power disturbance at 1 th peripheral element on the length of its transmission line and its power. The dependence is shown in Figure 6. One can see that for each value of the power of a generator, $P_{g}$, there is some critical length of the transmission line. Below this critical length the magnitude of the safe power is proportional to the power of a generator, while above critical length it aproaches to zero and the synchronous mode of the power grid is broken even by a weak power disturbance. Thus, choosing the connection length less than the critical one, we can greatly increase the stability of the 1 st element to the power disturbance. Similar results obtained in [Menck et al., 2014] where the model of the North European power grid with homogeneous couplings strength was considered. It was shown that peripheral elements with low node degree have the lowest stability to power disturbances. However, our results show that the stability of peripheral elements to disturbances not only depends on the degree of the element, but also on the lengths of their connections to the rest of the grid.

\section{Conclusion}

We studied the dynamics of two models of power grids, namely model of the small hub cluster grid and model of the Nizhny Novgorod power grid. For small hub cluster grid partition of parameters plane into the areas corresponding to different modes of operation are obtained. For the Nizhny Novgorod power grid we studied stability of synchronous mode against power perturbations of its elements. We found that peripheral elements of the power grid can be strongly or weakly stable to power disturbances depending on the length of the connection. Probably this result can help to prognoze the elements of the power grids more vulnerable to power disturbance. We suppose to study this problem elsewhere.

\section{Acknowledgements}

This work was supported by the Russian Science Foundation (Project No. 19-12-00338).

\section{References}

Arinushkin, P. A. and Anishchenko, V. S. (2018). Analysis of synchronous modes of coupled generators, stability of dynamic modes. Izvestia VUZ. Applied Nonlinear Dynamics, 26(3):62-77.

Dmitrichev, A. S., Zakharov, D. G., and Nekorkin, V. I. (2017). Global stability of a synchronous regime in hub clusters of the power networks. Radiophysics and Quantum Electronics, 60(6):506-512.

Drfler, F. and Bullo, F. (2011). Topological equivalence of a structure-preserving power network model and a non-uniform kuramoto model of coupled oscillators. In 2011 50th IEEE Conference on Decision and Control and European Control Conference, pages 70997104.

Drfler, F. and Bullo, F. (2012). Synchronization and transient stability in power networks and nonuniform kuramoto oscillators. SIAM Journal on Control and Optimization, 50(3):1616-1642.

Drfler, F., Chertkov, M., and Bullo, F. (2012). Synchronization assessment in power networks and coupled oscillators. In 2012 IEEE 51st IEEE Conference on Decision and Control (CDC), pages 4998-5003.

Dwivedi, A. and Yu, X. (2013). A maximum-flowbased complex network approach for power system vulnerability analysis. IEEE Transactions on Industrial Informatics, 9(1):81-88.

Filatrella, G., Nielsen, A. H., and Pedersen, N. F. (2008). Analysis of a power grid using a kuramotolike model. The European Physical Journal B, 61(4):485-491.

Gorev, A. A. (1959). Transient Processes of a Synchronous Machine [in Russian]. Gosenergoizdat, Moscow.

Grzybowski, J. M. V., Macau, E. E. N., and Yoneyama, T. (2018). Power-Grids as Complex Networks: 
Emerging Investigations into Robustness and Stability, pages 287-315. Springer International Publishing, Cham.

Hill, D. J. and Guanrong Chen (2006). Power systems as dynamic networks. In 2006 IEEE International Symposium on Circuits and Systems, pages 4 pp. -725 .

Klinshov, V. V., Nekorkin, V. I., and Kurths, J. (2015). Stability threshold approach for complex dynamical systems. New Journal of Physics, 18(1):013004.

Lozano, S., Buzna, L., and Díaz-Guilera, A. (2012). Role of network topology in the synchronization of power systems. The European Physical Journal B, 85(7):231.

Menck, P. J., Heitzig, J., Kurths, J., and Joachim Schellnhuber, H. (2014). How dead ends undermine power grid stability. Nature Communications, 5(3969).

Ministry of Energy of the Russian Federation (2014). About approval of the scheme and the development program of the unified energy system of russia in 2012 2020. The order N495 of 1 August 2014.

Motter, A. E., Myers, S. A., Marian, A., and Takashi, N. (2013). Spontaneous synchrony in power-grid networks. Nature Physics, 9(3):191-197.

Pagani, G. A. and Aiello, M. (2013). The power gridas a complex network: A survey. Physica A: Statistical Mechanics and its Applications, 392(11):2688 2700.

Park, R. (1929). Two-reaction theory of synchronous machines: Generalized method of analysis - part i. Transactions of the AIEE, 48:716730.

Rosas-Casals, M. (2010). Power grids as complex networks: Topology and fragility. In 2010 Complexity in Engineering, pages 21-26.

Solé, R. V., Rosas-Casals, M., Corominas-Murtra, B., and Valverde, S. (2008). Robustness of the european power grids under intentional attack. Phys. Rev. E, 77:026102.

Song, Y., Hill, D. J., and Liu, T. (2017). Local stability of dc microgrids: A perspective of graph laplacians with self-loops. In 2017 IEEE 56th Annual Conference on Decision and Control (CDC), pages 26292634.

Witthaut, D. and Timme, M. (2012). Braess's paradox in oscillator networks, desynchronization and power outage. New Journal of Physics, 14(8):083036.
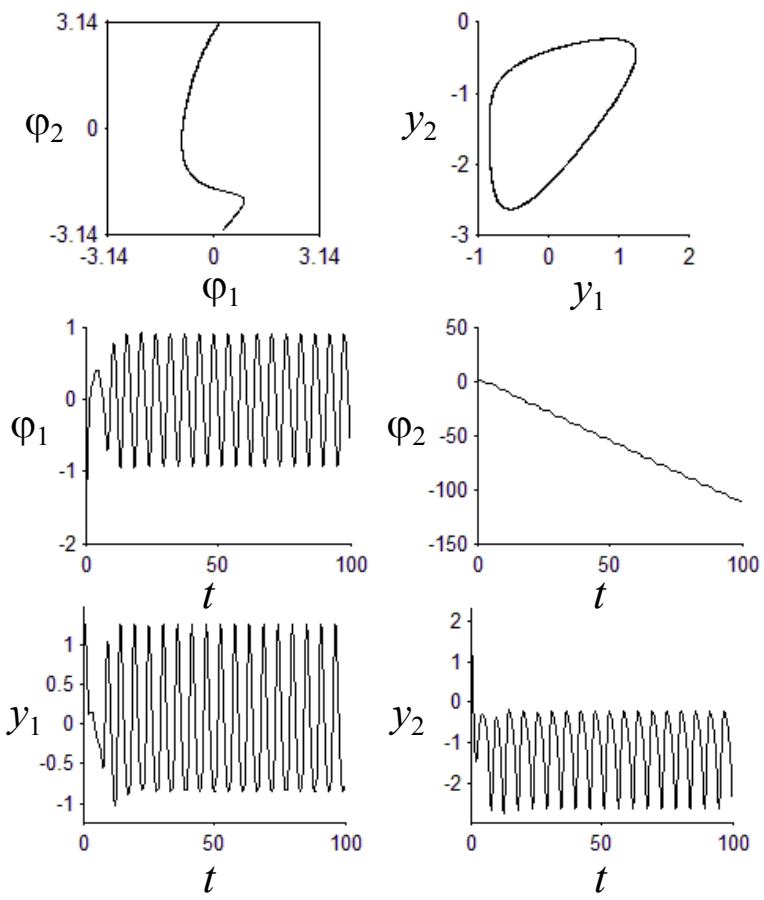

(a)
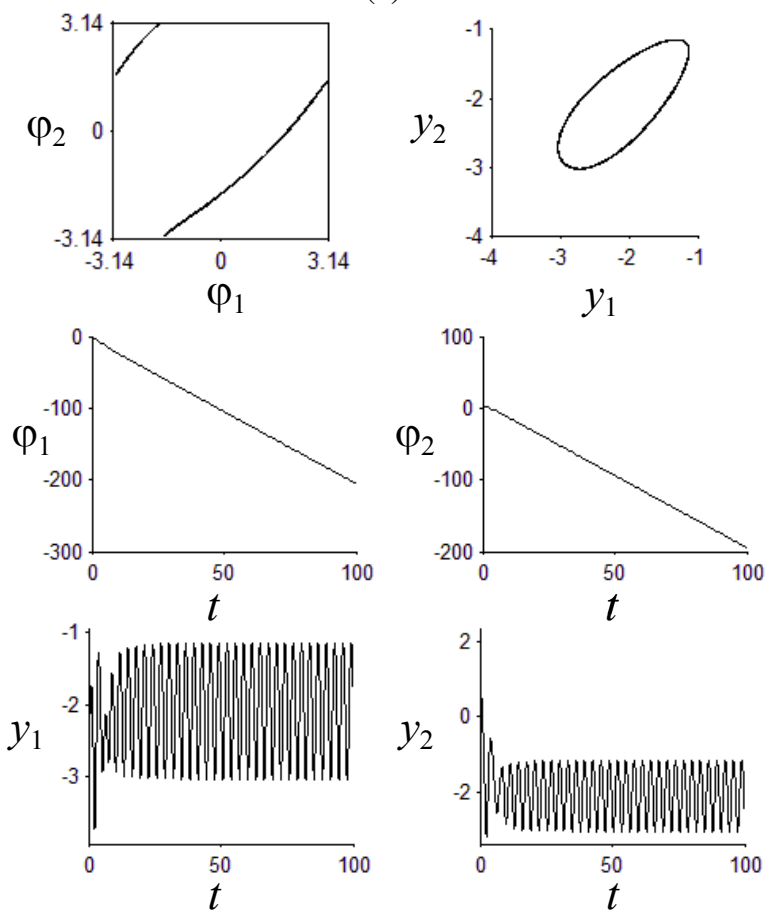

(b)

Figure 3. Phase portraits and waveforms corresponding to (a) quasi-synchronous and (b) asynchronous modes of the small hub cluster power grid model. Parameter values: $\lambda=0.7$ (a) $\gamma_{1}=$ $0.3, \gamma_{2}=1.5$ (b) $\gamma_{1}=1.7, \gamma_{2}=1.6$. 


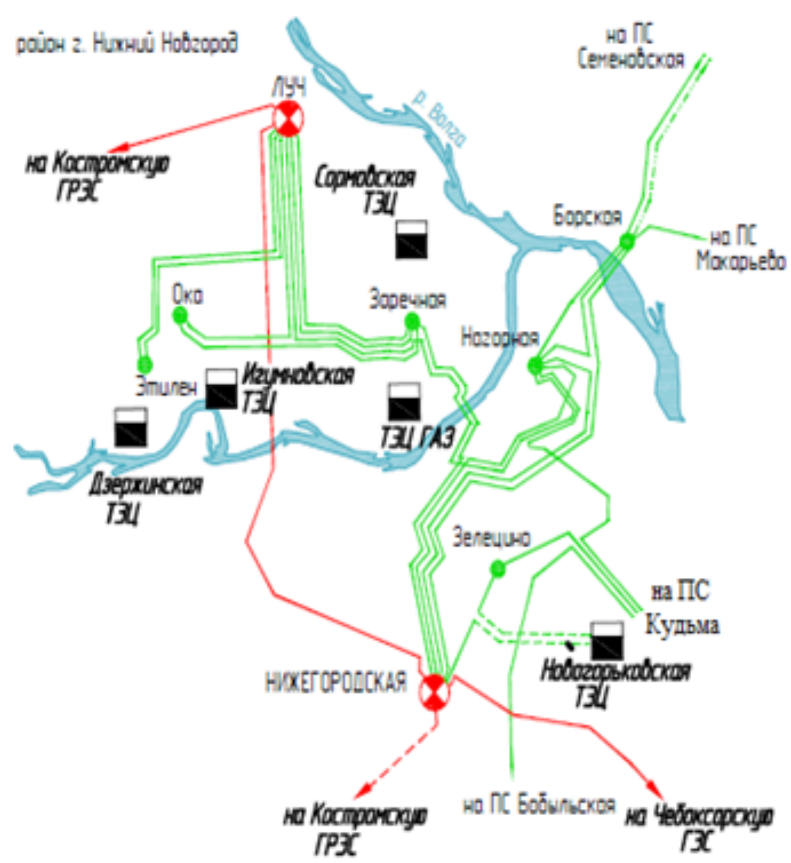

(a)

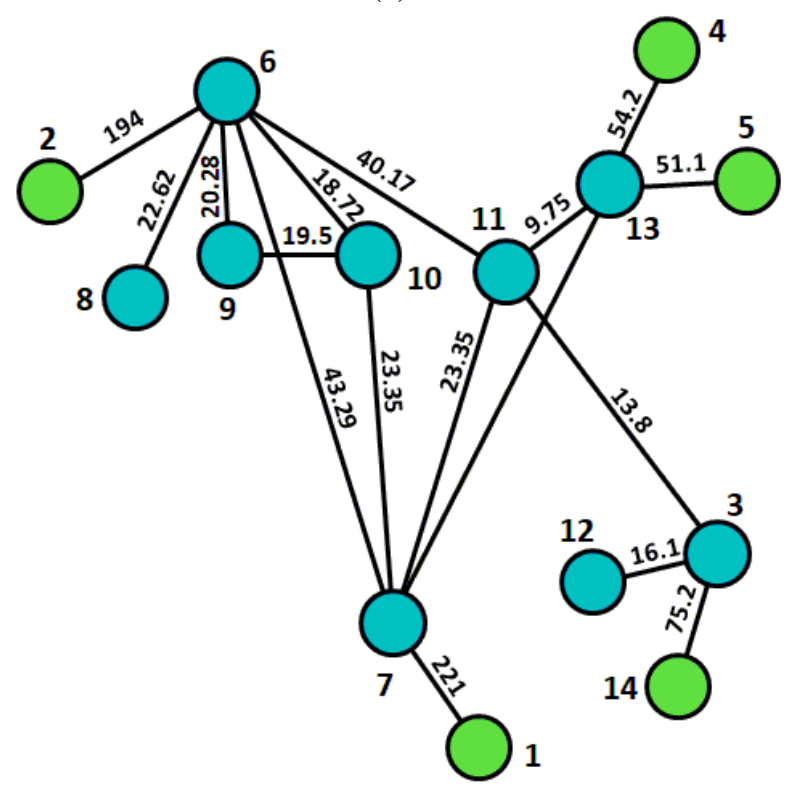

(b)

Figure 4. (a) Schematic layout of power lines and substations with a voltage from $220 \mathrm{kV}$ and above in Nizhny Novgorod (adopted from [Ministry of Energy of the Russian Federation, 2014] and (b) corresponding topology of the Nizhny Novgorod power grid model. Blue circles are consumers, green circles are generators. The lengths of the transmission lines in $\mathrm{km}$ are shown on the edges of the graph.

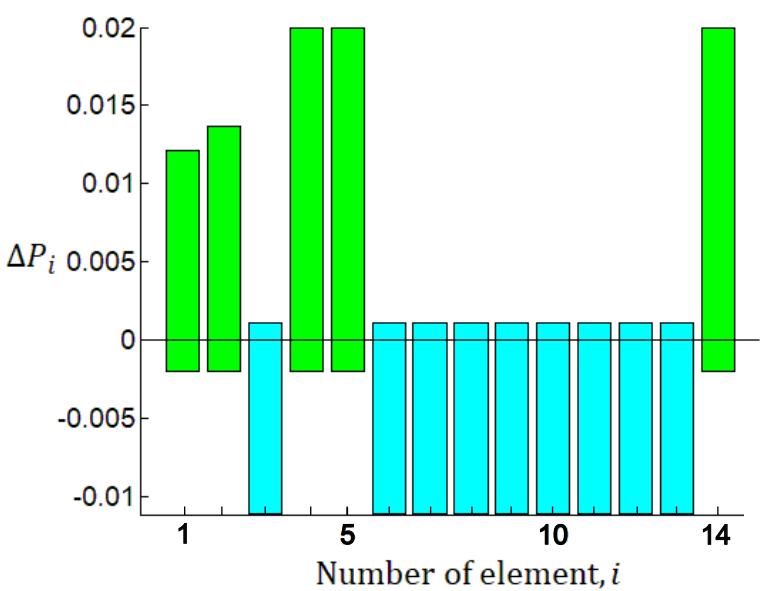

Figure 5. The magnitudes of safe power disturbances for consumers (blue) and generators (green) in the Nizhny Novgorod power grid model. Parameter values: $\tau=10, \alpha=0.02, P_{g}=$ 0.002 .

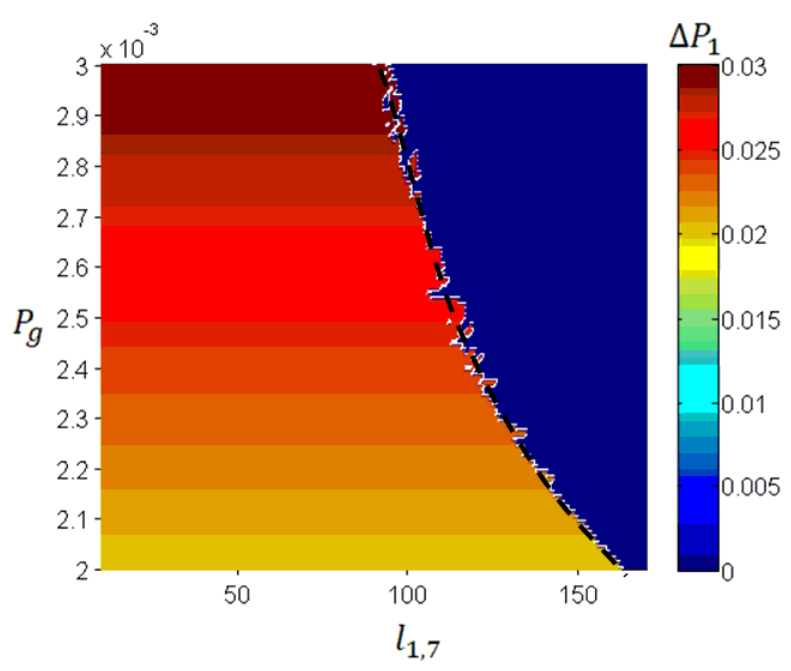

Figure 6. Diagram of the maximal safe power disturbance of 1th element in the Nizhny Novgorod power grid model. The dashed line indicates the critical length of the transmission line. Parameter values: $\tau=100, \alpha=0.02$. 